\title{
Sacral Erector Spinae Plane Block for Gender Reassignment Surgery
}

\author{
Promil Kukreja ${ }^{1}$, Paige Deichmann ${ }^{1}$, John P. Selph ${ }^{2}$, John Hebbard ${ }^{1}$, Hari Kalagara ${ }^{1}$
}

1. Anesthesiology and Perioperative Medicine, University of Alabama at Birmingham, Birmingham, USA 2. Urology, University of Alabama at Birmingham, Birmingham, USA

Corresponding author: Promil Kukreja, pkukreja@uabmc.edu

\begin{abstract}
The erector spinae plane block (ESPB) is an interfascial plane block that has been used to provide perioperative analgesia for a variety of indications. This case report describes the novel use of the sacral ESPB on a transgender patient undergoing male-to-female gender reassignment surgery for perioperative pain control. The sacral ESPB technique was described and post-operative pain score and opioid requirements were reported. The sacral ESPB was successfully used as an alternative to neuraxial, caudal, or peripheral nerve blocks for gender reassignment surgery.
\end{abstract}

Categories: Anesthesiology, Pain Management, Urology

Keywords: truncal block, regional anesthesia, interfascial plane block, penectomy, erector spinae plane block, gender reassignment surgery, multimodal analgesia, transgender, esp

\section{Introduction}

The erector spinae plane block (ESPB) is a novel paraspinal plane block first described in 2016 to relieve thoracic pain [1]. Since that time, the ESPB has been used for a number of novel indications and has been described in over 200 case reports [2]. Randomized controlled trials have shown the ESPB to be an effective perioperative analgesic for several thoracic procedures, including mastectomy, video-assisted thoracoscopy (VATS), and cardiac surgery [3-6]. At our institution, we regularly utilize the thoracic ESPB as a component of our regional anesthesia pain service for both chest and abdominal surgeries. More recently, the lumbar ESPB has been described for abdominal surgeries, prostatectomy, lumbar spine surgery, total hip arthroplasty, and proximal femur surgery [7-12].

Tulgar et al. were the first to describe the sacral ESPB as a means to provide analgesia to sacral dermatomes for a pilonidal sinus surgery [12].

After reading the aforementioned sacral ESPB case reports, we proceeded with the sacral ESPB for a novel indication. Using the midline approach described by Aksu et al., we performed the sacral ESPB on a patient undergoing gender reassignment surgery for perioperative pain control [13]. The sacral ESPB has since been

Received 04/02/2020 Review began 04/07/2020 Review ended 04/09/2020 Published 04/14/2020

\section{() Copyright 2020}

Kukreja et al. This is an open access article distributed under the terms of the Creative Commons Attribution License CC-BY 4.0., which permits unrestricted use, distribution, and reproduction in any medium, provided the original author and source are credited. described to provide pain control for pediatric hypospadias repair and lower extremity radicular pain [14,15].

The number of individuals in the US who carry a diagnosis of either gender identity disorder or transsexual has increased by over three fold from 2000 to 2014 and continues to increase [16]. Of those individuals, approximately 20\%-40\% seek gender reassignment surgery [16]. The incidence of gender reassignment surgery will likely rise as societal barriers, such as social stigma, access to care, and insurance coverage, are overcome. As these procedures become more common, anesthesiologists should seek out modalities to address perioperative pain.

\section{Case Presentation}

A 39-year-old American Society of Anesthesiologists (ASA) 2 transwoman, with a weight of $98.9 \mathrm{~kg}$ and height of $177 \mathrm{~cm}$ (body mass index (BMI) $31.3 \mathrm{~kg} / \mathrm{m} 2$ ), presented for gender reassignment surgery. Patient's gender reassignment surgery would include a total penectomy, bilateral orchiectomy, complete scrotectomy, anterior urethroplasty, construction of artificial vagina with graft, perineoplasty, clitoroplasty, and cystoscopy. A preoperative sacral ESPB for pain control was planned as part of a multimodal pain regimen. The morning of the surgery, the patient was placed in prone position with standard monitoring and given 1 mg midazolam and $50 \mathrm{mcg}$ fentanyl for sedation. Under aspetic precautions, a high-frequency linear ultrasound transducer was placed midline just above the sacrum.

Median sacral crests and erector spinae muscle/plane (ESP) were identified as described by Aksu et al. A 5 $\mathrm{cm}, 21 \mathrm{G}$ echogenic needle was inserted using an in-plane technique from cranial to caudal direction and advanced to the $\mathrm{S} 4$ crest (Figure 1). Following negative aspiration, $20 \mathrm{~mL}$ of $0.2 \%$ ropivacaine was injected at the S4 median sacral crest level with aspiration every $5 \mathrm{mLs}$. The process was repeated at the S2 median sacral crest level with an additional $20 \mathrm{~mL}$ of $0.2 \%$ ropivacaine (Figure 2). Local anesthetic (LA) was 


\section{Cureus}

deposited in the appropriate fascial plane between the erector spinae muscles and the S4 and S2 median sacral crests.

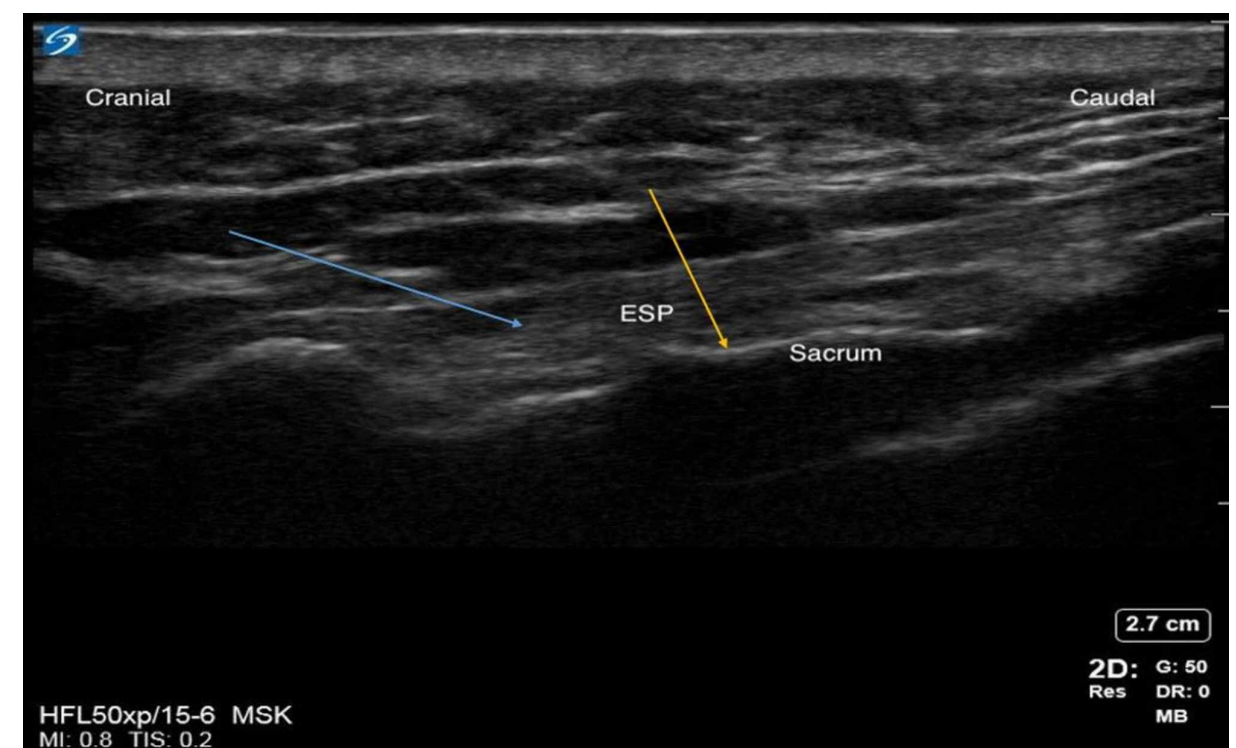

\section{FIGURE 1: Sacral erector spinae plane}

Blue arrow indicates the erector spinae plane. Yellow arrow indicates the sacrum.

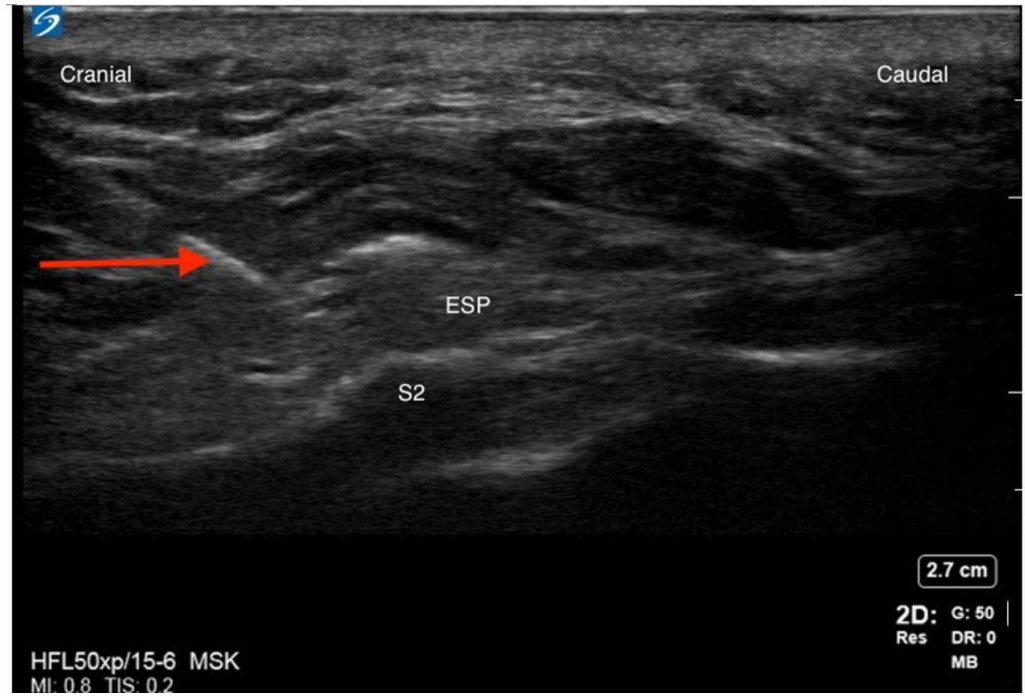

\section{FIGURE 2: S2 median sacral crest and needle path (red arrow)}

The red arrow indicates the needle.

The patient was then taken to the operating room for surgery under general anesthesia. The surgery lasted six hours and 20 minutes with total intraoperative opioid consumption of $150 \mathrm{mcg}$ of fentanyl for the entire procedure. After surgery, the patient was transported to post-anesthesia care unit (PACU) where she rated her post-operative pain as moderate (numerical rating scale $4-6$ ). The post-operative pain scores are shown in Figure 3. 


\section{Cureus}

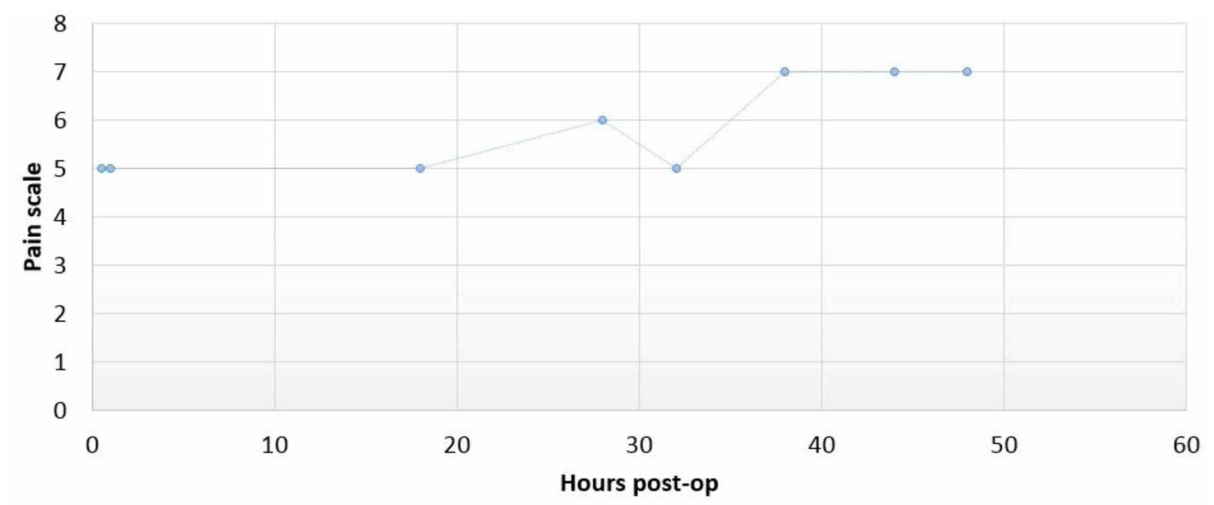

FIGURE 3: Pain score over time

Patient's complete post-operative pain regimen consisted of scheduled gabapentin $100 \mathrm{mg}$ TID, acetaminophen $650 \mathrm{mg} \mathrm{q6}$, ibuprofen $600 \mathrm{mg}$ TID and PRN oxycodone 5-10 mg and PRN IV hydromorphone $0.2 \mathrm{mg}$ for breakthrough pain. Daily oral morphine equivalents (OMEs) are listed below in Figure 4. Over the course of her hospitalization, she never required IV hydromorphone for breakthrough pain control.

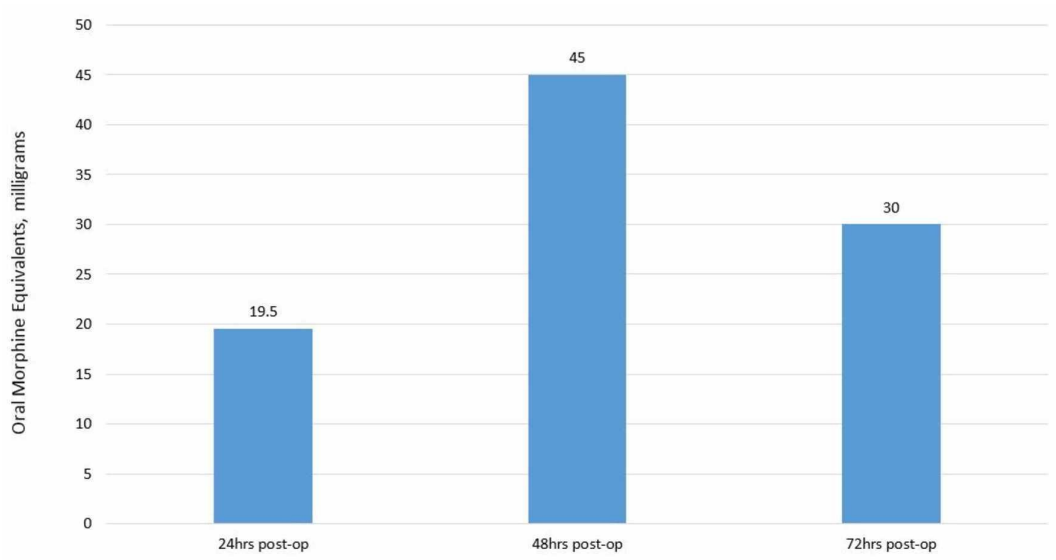

FIGURE 4: Post-operative oral morphine equivalents (OMEs) over time

\section{Discussion}

Gender-reassignment surgery was a challenging case for peri-operative analgesia to effectively control pain. There is evidence to support the use of neuraxial blocks to control post-operative pain, but we had some reservations based on the associated adverse effects with neuraxial blocks like hypotension. Also, postoperative anticoagulation regimen after neuraxial block may increase the risk of rare but dangerous adverse effects such as epidural hematoma. The location of the surgery and expected area of pain distribution precluded the consideration of transversus abdominis plane (TAP), quadratus lumborum, or lumbar plexus blocks. Pudendal nerve blocks would have enabled analgesia, but the risk of nerve injury due to close approximation to the surgical site and the expertise of regional anesthesia staff being comfortable in doing these bilateral pudendal nerve blocks moved us away from this option. Also, these nerve blocks would not have covered the affected area completely if used individually. As an alternative, we decided to proceed with a sacral ESP block.

ESPB is a relatively new regional interfacial plane block that provides somatic and visceral analgesia blocking the dorsal and ventral rami of spinal nerve along with the rami communicantes that transmit autonomic fibers to and from the sympathetic ganglia. Branches of the dorsal ramus innervates the skin of the back and branches of the ventral ramus supply lateral and anterior wall. ESPB has extensive craniocaudal spread of LA and helpful in blocking multiple dermatomes [16].

ESPB has potential applications where risk of using epidural outweighs benefits. ESPB block at sacral levels can potentially block pudendal nerve (S2-S4), and may also block part of lumbar plexus via cephalad spread. There is no other individual nerve block besides neuraxial block that can cover both perineum and genitalia. The innervation of external genitalia is mainly by pudendal nerve which arises from S2-S4 levels 
and accompany the pudendal vessels. Two more nerves ilioinguinal (L1) and genital branch of genitofemoral nerve (L1-L2) arise from lumbar plexus to innervate regions of external genitalia. The sacral ESPB offers a practical alternative to neuraxial block or caudal block to provide analgesia for such urological procedure.

The patient in this case report received very minimal opioids intraoperatively and also in the early postoperative period along with moderate pain scores until the block effect wore off. The midline technique offers single injection rather than transverse approach which requires bilateral injections to perform sacral ESP block. We preferred to do sacral ESP at 2 levels in this case at S4 and S2 levels with the aim of covering the pudendal innervation from S4 injection and to cover lower lumbar levels from S2 injection. The Sacral ESP block has not been described before for urological procedures like gender re-assignment. The ESP block has shown to effectively treat chronic thoracic pain, acute post-thoracotomy pain, breast surgery, as well as providing abdominal analgesia. There is minimal literature describing the use of ESP block for adult urological procedures, although there is some evidence for pediatric urological procedures. This is a novel technique described for the first time for these gender reassignment surgeries using a 2 level sacral ESP block. Most of these patients undergo repeated minor interventions in the early postoperative period which are quite painful and the potential for sacral ESP catheter to enable prolonged analgesia during the hospital stay is another likely advantage with this block in these surgeries.

The limitations are this is just one case report and we need further studies to validate the analgesic benefits of sacral ESP for these surgeries. We could not assess the sensory distribution of the block due to time constraints and there was no lower extremity motor weakness in the post-operative period. There is no clarity over the anatomical nomenclature for these sacral interfascial plane blocks currently as suggested by Hamilton et al. and proposed for sacral retrolaminar block as an alternative name [17]. Piracinni et al. proposed the name of sacral multifidus plane block instead of retrolaminar or ESP nomenclature $[13,14,18$ 20]. We suggest using any of the above nomenclatures as each of them are identical but with different proposed anatomical names. The sacral ESP block can offer many advantages, which were relevant to our patient. First, it provides coverage of multiple dermatomal levels by a longitudinal midline injection technique. Second, it is a superficial block and can be safely performed under ultrasound guidance. Third, it does not cause significant motor weakness. Fourth, ESP block may not cause significant hypotension and more hemodynamically stable compared to neuraxial blocks. Fifth, the potential to place the sacral ESP catheter to provide prolonged post-operative analgesia. These unique characteristics clearly favored sacral ESP block over other blocks like neuraxial, caudal, lumbar plexus, quadratus lumborum or pudendal nerve blocks

\section{Conclusions}

The sacral ESP (sacral retrolaminar or sacral multifidus plane) block is an alternative regional analgesic technique for urological procedures including gender reassignment procedures. It can be used as a viable alternative to neuraxial, caudal blocks and peripheral nerve blocks (pudendal nerve block) for these unique surgeries in this patient population. The efficacy of the sacral ESP blocks is not well established yet, randomized controlled trials are warranted for better understanding of the sacral ESP blocks with respect to analgesic efficacy and adverse effects. There needs to be a consensus on the nomenclature going forward for these sacral interfascial plane blocks to be adopted universally.

\section{Additional Information \\ Disclosures}

Human subjects: Consent was obtained by all participants in this study. Conflicts of interest: In compliance with the ICMJE uniform disclosure form, all authors declare the following: Payment/services info: All authors have declared that no financial support was received from any organization for the submitted work. Financial relationships: All authors have declared that they have no financial relationships at present or within the previous three years with any organizations that might have an interest in the submitted work. Other relationships: All authors have declared that there are no other relationships or activities that could appear to have influenced the submitted work.

\section{References}

1. Forero M, Adhikary SD, Lopez H, Tsui C, Chin KJ: The erector spinae plane block: a novel analgesic technique in thoracic neuropathic pain. Reg Anesth Pain Med. 2016, 41:621-627.

2. Curb your enthusiasm: Erector spinae plane block - 'because it is easy' is not a good reason to do it! . (2019). Accessed: March 1, 2020: https://www.asra.com/asra-news/article/213/curb-your-enthusiasm-erectorspinae-plan.

3. Singh S, Kumar G, Akhileshwar: An ultrasound-guided erector spinae plane block for postoperative analgesia in modified radical mastectomy: a randomised control study. Indian J Anaesth. 2019, 63:200-204.

4. Gaballah KM, Soltan WA, Bahgat NM: Ultrasound-guided serratus plane block versus erector spinae block for postoperative analgesia after video-assisted thoracoscopy: a pilot randomized controlled trial. J Cardiothorac Vasc Anesth. 2019, 33:1946-1953.

5. Krishna SN, Chauhan S, Bhoi D, Kaushal B, Hasija S, Sangdup T, Bisoi AK: Bilateral erector spinae plane block for acute post-surgical pain in adult cardiac surgical patients: a randomized controlled trial. J Cardiothorac Vasc Anesth. 2019, 33:368-375. 10.1053/j.jvca.2018.05.050 
6. Alttparmak B, Toker MK, Uysal AI, Kuscu Y, Demirbilek SG: Ultrasound-guided erector spinae plane block versus oblique subcostal transversus abdominis plane block for postoperative analgesia of adult patients undergoing laparoscopic cholecystectomy: randomized, controlled trial. J Clin Anesth. 2019, 57:31-36.

7. Tulgar S, Senturk O: Ultrasound guided low thoracic erector spinae plane block for postoperative analgesia in radical retropubic prostatectomy, a new indication. J Clin Anesth. 2018, 47:4.

10.1016/j.jclinane.2018.02.013

8. Singh S, Choudhary NK, Lalin D, Verma VK: Bilateral ultrasound-guided erector spinae plane block for postoperative analgesia in lumbar spine surgery: a randomized control trial [Epub ahead of print]. J Neurosurg Anesthesiol. 2019,

9. Cesur S, Yayik AM, Ozturk F, Ahiskalioglu A: Ultrasound-guided low thoracic erector spinae plane block for effective postoperative analgesia after lumbar surgery: report of five cases. Cureus. 2018, 10:e3603. 10.7759/cureus.3603

10. Tulgar S, Senturk O: Ultrasound guided erector spinae plane block at L-4 transverse process level provides effective postoperative analgesia for total hip arthroplasty. J Clin Anesth. 2018, 44:68.

10.1016/j.jclinane.2017.11.006

11. Tulgar S, Selvi O, Senturk O, Ermis MN, Cubuk R, Ozer Z: Clinical experiences of ultrasound-guided lumbar erector spinae plane block for hip joint and proximal femur surgeries. J Clin Anesth. 2018, 47:5-6. 10.1016/j.jclinane.2018.02.014

12. Tulgar S, Senturk O, Thomas DT, Deveci U, Ozer Z: A new technique for sensory blockage of posterior branches of sacral nerves: ultrasound guided sacral erector spinae plane block. J Clin Anesth. 2019, 57:12930. 10.1016/j.jclinane.2019.04.014

13. Aksu C, Gurkan Y: Sacral erector spinae plane block with longitudinal midline approach: could it be the new era for pediatric postoperative analgesia?. J Clin Anesth. 2020, 59:38-39. 10.1016/j.jclinane.2019.06.007

14. Piraccini E, Antioco M, Maitan S: Ultrasound guided sacral erector spinae plane block: a useful tool for radicular pain treatment. J Clin Anesth. 2020, 59:11-12. 10.1016/j.jclinane.2019.06.011

15. Canner JK, Harfouch O, Kodadek LM, et al.: Temporal trends in gender-affirming surgery among transgender patients in United States. JAMA Surg. 2018, 153:609-616. 10.1001/jamasurg.2017.6231

16. Kalagara HK, Deichmann P, Brooks B, Nagi P, Kukreja P: T1 erector spinae plane block catheter as a novel treatment modality for pancoast tumor pain. Cureus. 2019, 11:e6092. 10.7759/cureus.6092

17. Hamilton DL: The erector spinae plane block: time for clarity over anatomical nomenclature . J Clin Anesth. 2020, 62:109699. 10.1016/j.jclinane.2020.109699

18. Piraccini E, Taddei S: Sacral multifidus plane block: the correct name for sacral erector spinae plane block . J Clin Anesth. 2020, 63:109754. 10.1016/j.jclinane.2020.109754

19. Voscopoulos C, Palaniappan D, Zeballos J, Ko H, Janfaza D, Vlassakov K: The ultrasound- guided retrolaminar block. Can J Anesth. 2013, 60:889-95. 10.1007/s12630-013-9983-X

20. Yamauchi M, Sato Y: Complex issues in new ultrasound-guided nerve blocks: how to name, where to inject, and how to publish. J Anesth. 2018, 32:283-7. 10.1007/s00540-018-2452-5 